\title{
Design of Energy Efficient Control Unit and Implementation on High Performance FPGA
}

\author{
Keshav Kumar, BishwajeetPandey, D. M. Akbar Hussian,Arifa Bhutto, Amit Kant Pandit, \\ Yousef A. Baker El-Ebiary
}

\begin{abstract}
With the crisis of power across the globe, green communication and power-efficient devices are getting more and more attention. This work emphasis about the implementation of Control Unit $(\mathrm{CU})$ circuit on FPGA kit. In this project, power consumption of $C U$ circuit is analyzed by changing the different Input/Output (I/O) standards of FPGA. This project is implemented on Xilinx 14.1 tool and the power consumption on $C U$ is calculated with X Power Analyzer tool on 28-Nano-Meter (nm) Artix-7 Field Programmable Gate Array (FPGA). Out of different $I / O$ standards, $C U$ circuit is most power efficient with LVCMOS I/O standard on Artix-7 FPGA.
\end{abstract}

Keywords: Control Unit, I/O standard, Low Power, LVCMOS, FPGA.

\section{INTRODUCTION}

With the increase in population and industrialization across the entire world, the world is facing a huge problem of energy and power crisis [1]. Green communication and power efficient devices are the most suitable technique for overcoming from the problem of energy and power crisis [2]. Keeping all these aspect in knowledge, this research work gives an idea about designing a power-efficient $\mathrm{CU}$ circuit with FPGA. The control unit is the basic and one of the initial components of Central Processing Unit (CPU) [3]. The function of CU is to respond on the set of instructions, which is to be sent to the processor of CPU. In this research work, various LVCMOS I/O standard [4] of Artix-7 FPGA which is shown in figure 1 is changed, to calculate the power utilization on $\mathrm{CU}$.

$\begin{array}{cccc} & & & \\ \text { LVCMOS15 } & \text { LVCMOS } & \text { LVCMOS } & \text { LVCMOS } \\ & 18 & 25 & 33\end{array}$

Figure 1.Different LVCMOS I/O standards.

\section{LVCMOS I/O Standards.}

The LVCMOS is low voltage digital integrated circuit of CMOS technology [5]. I/O standards are Input/Output standards, which works to match the impedance of device with input line, input port, output line and output port, in

Revised Manuscript Received on October 23, 2019.

Keshav Kumar, Gyancity Research Consultancy, Motihari, Bihar. India.(E-mail: thekeshavkumar1993@gmail.com)

BishwajeetPandey, Gyancity Research Consultancy, Motihari, Bihar. India.(E-mail: gyancity@gyancity.com)

D. M. Akbar Hussian, Aalborg University, Esbjerg, Denmark.(Email: akh@et.aau.dk)

Arifa Bhutto, University of Sindh, Jamshoro, Pakistan(E-mail: arifa.bhutto@usimdh.edu.pk)

Amit Kant Pandit, Shri Mata Vaishno Devi University (SMVDU), Katra, Jammu and Kashmir, India.(E-mail: amit.pandit@smvdu.ac.in)

Yousef A. Baker El-Ebiary, Faculty of Informatics and Computing, UniSZA, Malaysia(E-mail: yousefelebiary@unisza.edu.my) order to avoid the reflection of transmission line. With $28 \mathrm{~nm}$ Artix-7 FPGA, LVCMOS I/O standards is operated at maximum voltage of $3.3 \mathrm{~V}$. The different LVCMOS I/O standards are following:

- LVCMOS12

- LVCMOS15

- LVCMOS18

- LVCMOS25

- LVCMOS33

In this work, power analysis is done only for LVCMOS15, LVCMOS18, LVCMOS25 and LVCMOS33. LVCMOS12 is not compatible with 28nm Artix-7 FPGA.

\section{II.METHODOLOGY}

High-performance Random-Access Memory (RAM) design is implemented on $28 \mathrm{~nm}$ FPGA [6]. In this work power calculated for different LVCMOS I/O standards. Using LVCMOS I/O standards, authors have designed a low power D Flip-Flop [7]. Green data flip-flop design is implemented on FPGA using different LVCMOS I/O standards [8]. LVCMOS I/O standards based 4-bit register [9] is designed by authors on FPGA. Researcher also design Energy efficient digital clock with the help of LVCMOS I/O standards is implemented on Spartan-6 FPGA [10]. Clock gating-based energy efficient Arithmetic Logic Unit (ALU) design is implemented on FPGA by scaling operating frequency [11]. Therefore, a lot of work has been done on LVCMOS I/O standard-based FPGA devices for promoting green communication and power efficient devices. But no work is done on LVCMOS based CU circuit. So, in this work, a power efficient LVCMOS based Control Unit circuit is designed on 28-nm FPGA

\section{EXPERIMENTAL SECTION}

In this research work, the interfacing of $\mathrm{CU}$ with Artix-7 FPGA is done at an ambient temperature of $25^{\circ} \mathrm{C}$ with the airflow of 250 Linear Feet per Meter (LFM). The power is calculated at the frequency of $1 \mathrm{GHz}$. The power calculated for different LVCMOS I/O standard is analyzed by X Power Analyzer tool. The CU takes 8-bit data instruction, clock signal, reset signal and zero as input for the operation. The output of CU has 13 wire line. The Register-Transfer Level (RTL) schematic of the CU is presented in figure 2.

Published By: 


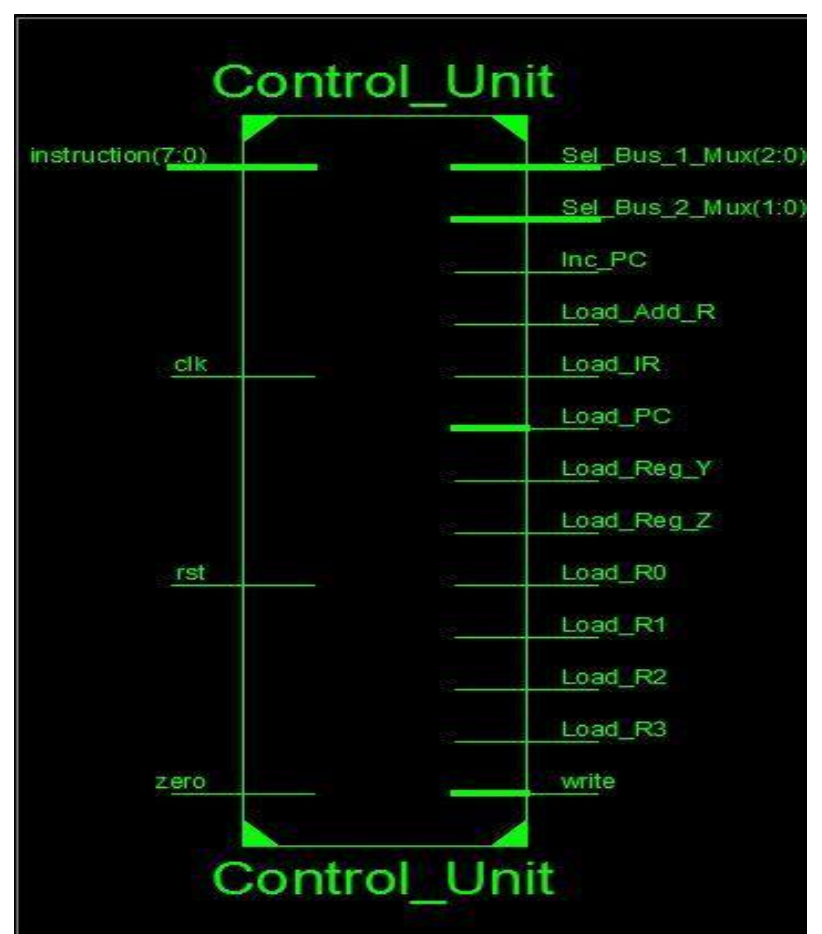

Figure 2.Schematic of Control Unit.

\section{POWER ANALYSIS.}

The total on-chip power dissipation is calculated for LVCMOS15, LVCMOS18, LVCMOS25 and LVCMOS33. The total consumption of on-chip power is sum up of static power and dynamic power. Static power is also called as leakage power of FPGA. Dynamic power is the sum of Clock power usage, Logic power dissipation, Signal power utilization and I/O power consumption of FPGA.

\section{A. Power Analysis of LVCMOS15.}

The total power of LVCMOS15 is $0.084 \mathrm{~W}$ which constitutes clock power utilization of $0.005 \mathrm{~W}$, logic power usage of $0.001 \mathrm{~W}$, signal power dissipation of $0.001 \mathrm{~W}, \mathrm{I} / \mathrm{O}$ power consumption of $0.036 \mathrm{~W}$ and leakage power dissipation of $0.042 \mathrm{~W}$. The power analysis is represented in figure 3 .

\section{Total On-Chip of LVCMOS15}

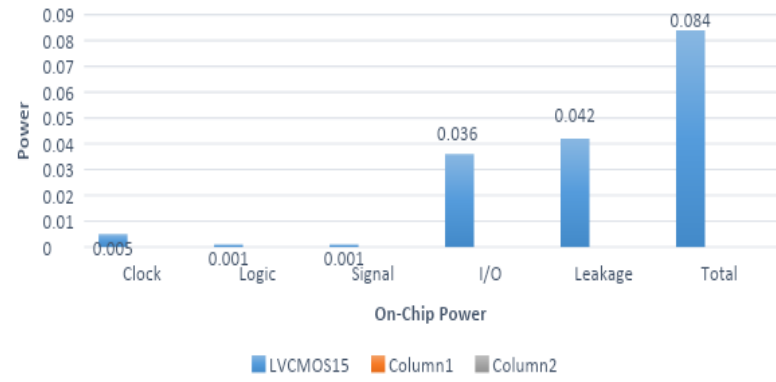

Figure 3.Power Analysis of LVCMOS15.

\section{B. $\quad$ Power Analysis OF LVCMOS18.}

For LVCMOS18 I/O standard, clock power dissipation is $0.005 \mathrm{~W}$, logic power utilization is $0.001 \mathrm{~W}$, signal power usage is $0.001 \mathrm{~W}, \mathrm{I} / \mathrm{O}$ power consumption is $0.048 \mathrm{~W}$, leakage power dissipation is $0.043 \mathrm{~W}$ and total power used by device is $0.097 \mathrm{~W}$, which is shown in Figure 4.

\section{Total On-Chip of LVCMOS18}

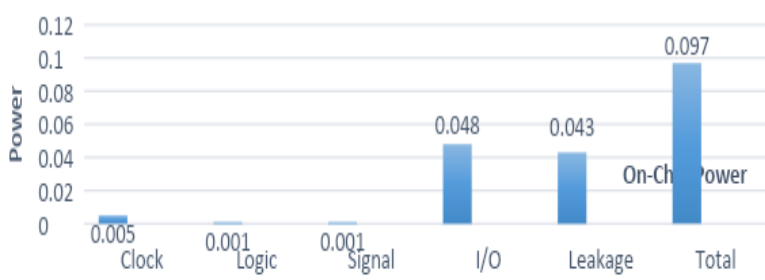

alvCMOS15 EColumn1 acolumn2

Figure 4.Power Analysis of LVCMOS18.

C.

Power Analysis OF LVCMOS25.

The total on-chip power of LVCMOS25 is described in Figure 5. The total power usage of the device is $0.135 \mathrm{~W}$, which is sum up of, clock power utilization of $0.005 \mathrm{~W}$, logic power usage of $0.001 \mathrm{~W}$, signal power utilization of $0.001 \mathrm{~W}, \mathrm{I} / \mathrm{O}$ power usage of $0.086 \mathrm{~W}$ and leakage power utilization of $0.043 \mathrm{~W}$.

\section{Total On-Chip of LVCMOS25}

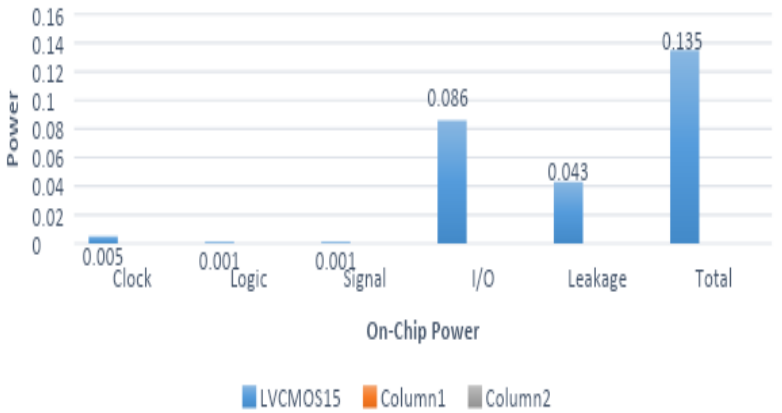

Figure 5.Power Analysis of LVCMOS25.

D. Power Analysis OF LVCMOS33.

In Figure 6, total on-chip power for LVCMOS33 is shown. For LVCMOS33 I/O standard clock power, logic power and signal power of device is same as for LVCMOS15, LVCMOS18 and LVCMOS25. For LVCMOS33, I/O power utilization is $0.146 \mathrm{~W}$, leakage power dissipation is $0.044 \mathrm{~W}$ and total power is $0.197 \mathrm{~W}$.

\section{Total On-Chip of LVCMOS33}

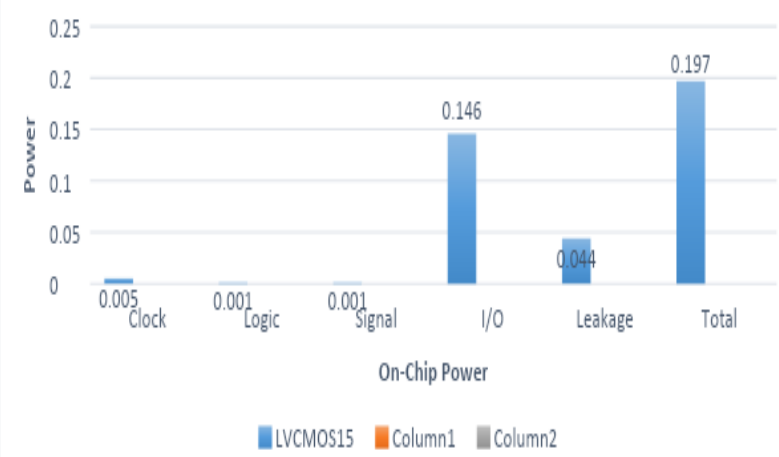

Figure 6.Power Analysis of LVCMOS33. 


\section{RESULT ANALYSIS}

From the power analysis of all above mentioned LVCMOS I/O standard, it is observed that total power dissipation of device increases when LVCMOS I/O standard is changed. LVCMOS15 I/O standard utilizes least amount of power and LVCMOS33 consumes the highest amount of power for device operation. The total power consumption of all the LVCMOS I/O standard is shown in Figure 7. LVCMOS18 utilizes $15.4762 \%$ more power, when compared with LVCMOS15 I/O standard. LVCMOS25 consumes $60.7143 \%$ more power than LVCMOS15 I/O standard. LVCMOS33 uses $134.524 \%$ more power, when compared with LVCMOS15 I/O standard.

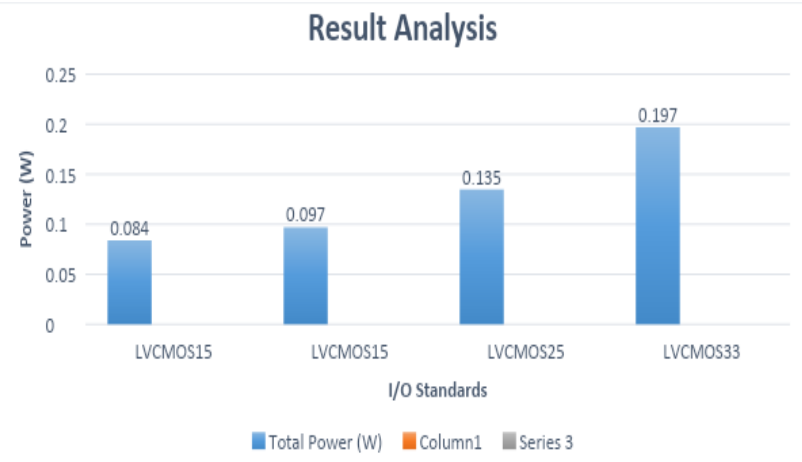

Figure 7.Total Power of LVCMOS I/O standards.

\section{CONCLUSION}

In this research work, the $\mathrm{CU}$ circuit is interfaced with 28-nm Artix-7 FPGA. For power calculation of the device, the I/O standard of FPGA is changed. It is observed that at $1 \mathrm{GHz}$ of frequency device is most power efficient with LVCMOS15 I/O standard. LVCMOS33 I/O standard uses highest amount of power, so this will not be beneficial for promoting green communication.

\section{REFERENCES}

1 www.conserve-energy-future.com/causes-and-solutionto-the-global-energy-crisis.php

2 R. Mahapatra, et.al. Energy efficiency tradeoff mechanism towards wireless green communication: A survey. IEEE Communications Surveys \& Tutorials, Vol. 18, Issue, 2015, pp: 686-705.

3 https://www.computerhope.com/jargon/c/contunit.htm

4 A. Aggarwal, et.al. LVCMOS-Based Low-Power Thermal-Aware Energy-Proficient Vedic Multiplier Design on Different FPGAs. In System and Architecture, Springer, Singapore, 2018.

5 L. Kalra, et.al. LVCMOS I/O standard based environment friendly low power ROM design on FPGA. In 2015 2nd International Conference on Computing for Sustainable Global Development (INDIACom), 2015.

6 S. Bhalla, T. Kaur, K. Bansal, I. Ahuja, and S. Chawla. LVCMOS IO Standard Based High Performance RAM Design on 28nm FPGA. International Journal of Control and Automation, Vol. 9, no. 9, pp. 213-220, 2016.

7 T. Agrawal, et.al. LVCMOS Based Energy Efficient D flip-flop Design. 2nd International Conference on ISMAC (IoT in Social, Mobile, Analytics and Cloud) (ISMAC), 2018.

8 G. Gupta, A. Kaur, and B. Pandey. LVCMOS based Green Data Flip Flop Design on FPGA. Ninth International Conference on Advanced Computing (ICoAC), pp. 41-45, 2017.
9 T. Agrawal, A. Kumar, P. Aggarwal, and S. S. Tirmizi. LVCMOS Based 4-Bit Register. 9th International Conference on Computing, Communication and Networking Technologies (ICCCNT), 2018.

10 S. Pandey, et. al. Low Power Digital Clock Design Using LVCMOS Input/Output Standards on $45 \mathrm{~nm}$ FPGA. Gyancity Journal of Engineering and Technology, Volume 2, Issue 2, 2016.

11 B. Pandey, et.al. Clock gating based energy efficient ALU design and implementation on FPGA. International Conference on Energy Efficient Technologies for Sustainability, 2013.

\section{AUTHORS PROFILE}

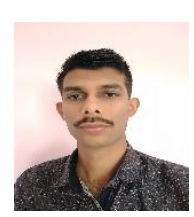

Keshav Kumar is pursuing his Master of Engineering in the field of Cyber Security and FPGA from Chitkara University, Punjab, India. He did his B.Tech in Electronics and Communication Engineering from Rajasthan Technical University, Kota, India. His area of interest is the implementation of security algorithms on FPGA, power-efficient device on FPGA and green communication. He has worked with researchers of three different countries in the field of Low Power VLSI design on FPGA and Energy Efficient devices for Green Communication.

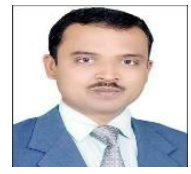

BishwajeetPandey is a co-founder of Gyancity Research Lab. Gyancity Research Lab organizes three conferences (ICGCET.ORG, RTCSE.ORG, IMCES.TECH) across the globe. He has completed his PhD in CSE from GSSI, Italy. $\mathrm{He}$ has worked as Asst. Professor in Department of Research at Chitkara University, Junior Research Fellow (JRF) at South Asian University. He has completed Master of Technology (IIIT Gwalior) in CSE with Specialization in VLSI, Master of Computer Application, R\&D Project in CDAC-Noida. He has authored and coauthored over 135 paper available on his Scopus Profile:https:// www.scopus.com/authid/detail.uri?authorId=57203239026. He has 1345 Citation according to his Google Scholar Profile: https://scholar.google.co.in/citations?user=UZ_8yAMAAAAJ\&hl=en

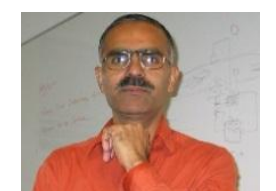

Dr. Dil Muhammad Akbar Hussain is a professor of Electronics Engineering \& Computer Science at the Department of Energy Technology, Aalborg University Denmark. Professor Akbar has a Master degree in Digital Electronics from Govt College University, Lahore Pakistan and a P-hD degree in Control Engineering from the School of Engineering and Applied Sciences, University of Sussex United Kingdom. Aalborg University has Two Satellite Campuses, one in Copenhagen (Aalborg University Copenhagen) and the other in Esbjerg (Aalborg University Esbjerg).

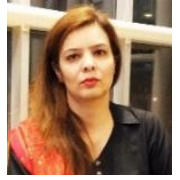

Arifa Bhutto works at IICT,University of Sindh Jamshoro, Pakistan. She is Experienced Assistant with a demonstrated history of working in the education management industry. Strong administrative professional skilled in Matlab, $\mathrm{C}++$ Lecturing, Algorithms, and Research. 
Processing and VDTN.

Dr. AMIT KANT PANDIT is an Associate

Professor in Department of Electronics and

Communication Engineering at Shri Mata Vaishno

Devi University Katra, India. He has teaching

experience of approx. 17 years. His area of interest

are Multimedia Technology, Digital Signal

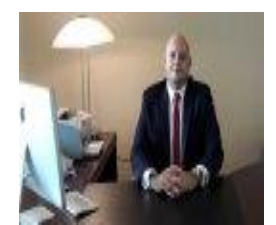

Assoc. Prof. Dr. YousefA.Baker El-

Ebiary.Holding Ph.D. in MIS, two Masters in IT

and Business administration, and high diploma

in Executive Management, Working at Faculty

of Informatics and Computing (FIK), Universiti

Sultan ZainalAbidin (UniSZA), Malaysia, as a

senior lecturer and member of several

committees.Over 11 years' experience in teaching levels of degree and

graduate studies. Also supervise both Ph.D.'s and Master students. A

member in many related associations such as IEEE, IAENG, ACSE,

IACSIT. 